\begin{abstract}
Rong Xue Wu • Martin Laser • Hong Han • Jeeva Varadarajulu • Kai Schuh • Matthias Hallhuber • Kai Hu • Georg Ertl · Christof R. Hauck • Oliver Ritter

\section{Fibroblast migration after myocardial infarction is regulated by transient SPARC expression}

\begin{abstract}
Secreted protein, acidic, and rich in cysteine (SPARC) is thought to regulate cell matrix interaction during wound repair. We hypothesized that SPARC might promote migration via integrin-dependent mechanisms. The present study was designed to clarify the contribution of SPARC in the wound healing process after myocardial infarction (MI). Adult mice received a specific $\alpha_{v}$ integrin inhibitor or vehicle through osmotic mini pumps. Mice of each group were either sham-operated or MI was induced. SPARC expression was investigated 2 days, 7 days, and 1 month after the surgical procedure. For migration assays, a modified Boyden chamber assay was used. A transient increase of SPARC levels was observed, starting at day 2 $(2.55 \pm 0.21)$, day $7(3.72 \pm 0.28)$, and 1 month $(1.9 \pm 0.16)$ after MI. After 2 months, SPARC expression dropped back to normal levels compared to sham-operated hearts. Immunofluorescence analysis showed an increase of SPARC in the infarcted area 2 days after MI, a strong increase in the scar area 7 days after MI, and only low levels in the scar area 2 months after MI. Integrin $\alpha_{v}$ inhibition abolished the up-
\end{abstract}

Electronic Supplementary Material Supplementary material is available for this article at http://dx.doi.org/10.1007/s00109-0050026-0.

R. X. Wu · M. Laser $\cdot$ H. Han · M. Hallhuber

K. Hu $\cdot$ G. Ertl $\cdot$ O. Ritter $(\bowtie)$

Department of Medicine I, Center for Cardiovascular Medicine, University of Würzburg,

Josef-Schneider-Str. 2,

97080 Würzburg, Germany

e-mail: Ritter_O@klinik.uni-wuerzburg.de

Tel.: +49-931-36112

Fax: +49-931-36212

J. Varadarajulu · C. R. Hauck

Zentrum für Infektionsforschung, University of Würzburg,

Würzburg, Germany

K. Schuh

Institute for Clinical Biochemistry, University of Würzburg,

Würzburg, Germany

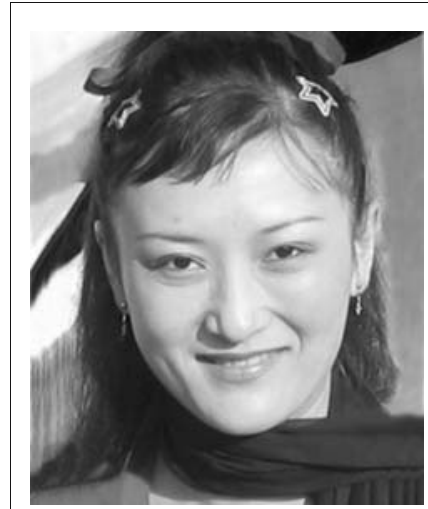

RonGXue Wu

has been working as M.D. in China. There she focused on clinical research in infectious diseases. She is currently a $\mathrm{Ph}$. D. student at the University of Würzburg, Germany and is working in the lab group of molecular cardiology. Her research interests are gene expression profiling, extracellular matrix proteins, and integrin signaling in wound healing processes of the heart.

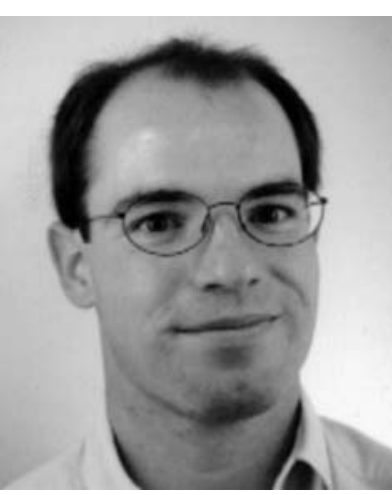

OLIVER RitTER went to medical school at the University of Heidelberg and at the University of Texas. After working for four years at the Franz Volhard Klinik and the Max Delbrück Center, he established his research group at the University of Würzburg. He is currently working as a cardiologist/electrophysiologist at the Department of Medicine I. His research focus is clinical electrophysiology and intracellular signaling in myocardial hypertrophy and failure. regulation of SPARC. In vitro migration assays demonstrated that fibronectin-stimulated haptotaxis of fibroblasts was modulated by SPARC. This study provides evidence that SPARC is significantly up-regulated in the infarcted region after MI. This up-regulation is dependent on $\alpha_{v}$ integrins. As SPARC is found to regulate fibroblast migration, it appears to play an important role in the injured myocardium with regard to healing and scar formation.

Keywords Myocardial infarction · Tissue remodeling · Extracellular matrix $\cdot$ Cell migration 


\section{Introduction}

Following myocardial infarction, the injured heart tissue undergoes a process of wound healing and scar formation, which is necessary to maintain structural and functional integrity of the organ. The preservation of cardiac function partly depends on the invasion of cardiac fibroblasts and restructuring of the extracellular matrix (ECM). In particular, deposition of ECM proteins such as collagen, fibronectin $(\mathrm{FN})$, and vitronectin (VN) at the infarcted area is thought to replace necrotic myocytes and leads to scar formation. The main family of cell surface receptors that sense the composition and structure of the ECM is the integrins [1].

In addition to classical ECM proteins, a number of socalled matricellular proteins have been described. Their role in cell adhesion and ECM remodeling is less welldefined, but they seem to act as modulators of cell-ECM interactions. Prototype examples of matricellular proteins include thrombospondin (TSP)-1,TSP-2, tenascin C, osteopontin, and also secreted protein, acidic, and rich in cysteine (SPARC) [2]. Studies in SPARC knockout mice indicate that these proteins influence the wound healing response and play critical regulatory roles in tissue renewal [3]. SPARC, also known as osteonectin or BM-40, is an extracellular $\mathrm{Ca}^{2+}$-binding glycoprotein that promotes deadhesion of cells from the matrix and influences migration, proliferation, shape, and motility of cultured cells [4]. Recent studies have also indicated an increased expression of SPARC in the myocardium after injury or stress [5-7].

High levels of SPARC expression are observed during tumor growth [8] and after tissue injury [9]. SPARC is able to promote prostate cancer cell migration, and this activity of SPARC is functionally connected to integrins $\alpha_{v} \beta_{3}$ and $\alpha_{\mathrm{v}} \beta_{5}$ [10]. SPARC protein induction through $\beta_{3}$-integrin expression has been reported [11]. These findings suggest that cell migration during tumor growth or wound repair is influenced by an interplay between integrins, including $\alpha_{\mathrm{v}}$ and the matricellular protein SPARC. However, the potential role of SPARC in the myocardium is still speculative, and how SPARC expression and function are integrated into the ECM remodeling events following myocardial infarction is currently unknown.

In the present study, we investigated altered gene expression of ECM proteins and SPARC in response to experimental myocardial infarction in the mouse. We followed the hypothesis that SPARC expression might be induced by integrins and that increased SPARC levels may account for scar formation after myocardial infarction via fibroblast invasion.

\section{Materials and methods}

Animal model

Adult male and female $\mathrm{C} 57 \mathrm{Bl} / 6$ mice weighing 22-25 g at the beginning of the study were used. Myocardial infarc- tion was induced as previously described under anesthesia by ligation of the descending branch of the left coronary artery. The overall mortality was $38 \%$ in the infarction group. Sham-operated animals underwent the same procedure except that no ligation was performed. No mortality was observed in this group.

\section{RNA isolation and cDNA array analysis}

Total RNA was extracted from the tissue of individual mouse hearts from five or six animals per treatment and time point using TRIzol (Invitrogen, Karlsruhe, Germany) according to the manufacturer's instructions. Following phenol/chloroform extraction, RNA quality was evaluated by spectrophotometry and formaldehyde agarose gel electrophoresis. RNA $(5 \mu \mathrm{g})$ was used for reverse transcription with gene-specific primers in the presence of biotin-dUTP using the Ampolabeling LPR kit (SuperArray, Frederick, MD). Biotinylated cDNA probes were denatured, and individual samples derived from each animal of the different treatment groups were hybridized to the GEArray Q series cDNA array "Extracellular matrix and adhesion molecules" (SuperArray) at $60^{\circ} \mathrm{C}$ for $17 \mathrm{~h}$. The GEArray membranes were then washed and blocked with GEA blocking solution, incubated with alkaline phosphatase-conjugated streptavidin, washed twice with $2 \times$ saline sodium citrate buffer (SSC) containing 1\% sodium dodecyl sulfate (SDS) and then twice with $0.1 \times \mathrm{SSC} / 1 \% \mathrm{SDS}$ at $60^{\circ} \mathrm{C}$ for $15 \mathrm{~min}$ each. Chemiluminescent detection was performed after incubation of the membranes with CDP-Star substrate (SuperArray). The results were analyzed using ScanAlyze2 software (M. Eisen, Stanford, CA) and the GEArray Expression Analysis Suite (SuperArray). Macroarray experiments were performed twice with each individual cDNA sample. In addition to the 96 gene-specific spots, the arrays contained positive [beta-actin, cyclophilin A, glyceraldehyde-3-phosphate dehydrogenase (GAPDH), ribosomal protein L13a] and negative (bacterial pUC18 plasmid) controls that were functional in each hybridization (for a complete list of genes and their localization on the array, see Supplementary Table 1). Before individual experiments were compared, the signals derived from GAPDH were used to normalize the signal intensity of each membrane. Intensity differences of more than 1.8-fold were considered significant and are presented in Table 1.

Tissue lysis and Western blot analysis

Mouse heart tissue was homogenized in 2\% Tris-Triton buffer. Following electrophoresis, the proteins were transferred to polyvinylidene fluoride membranes (Invitrogen). Anti-SPARC antibodies (biotinylated, Cat. no. BAF941, R\&D System, Minneapolis, USA) were used for detection of SPARC in Western blots. Blots were developed by enhanced chemiluminescence, and signals were detected by a digital imaging system (Chemilmager 5500, ALPHA 
Table 1 Differential expression of genes in mouse hearts 2 and 7 days after experimental myocardial infarction (MI)
The expression levels are shown as fold increase compared to sham-operated samples after normalization using the internal control (GAPDH). Values represent mean \pm SEM of independent hybridizations of RNA isolated from five to six animals for each treatment for each time point

${ }^{\mathrm{a}}$ The listed genes displayed on average a more than 1.8 -fold difference between the shamoperated and the infarcted animals 2 days and/or 7 days after the intervention

\begin{tabular}{|c|c|c|c|}
\hline \multirow[t]{2}{*}{ Spot location } & \multirow[t]{2}{*}{ Gene name } & \multicolumn{2}{|c|}{ Fold induction (MI vs sham-operated) } \\
\hline & & Day $2(n=6)$ & Day $7(n=5)$ \\
\hline \multicolumn{4}{|c|}{ Cell adhesion molecules } \\
\hline 17 & CEACAM 1 & $1.76 \pm 0.47$ & $4.73 \pm 0.51^{\mathrm{a}}$ \\
\hline 36 & Integrin alpha 4 & $1.50 \pm 0.40$ & $6.90 \pm 1.97^{\mathrm{a}}$ \\
\hline 38 & Integrin alpha 6 & $0.38 \pm 0.17$ & $2.03 \pm 0.30^{\mathrm{a}}$ \\
\hline 39 & Integrin alpha 7 & $1.88 \pm 0.26^{\mathrm{a}}$ & $3.69 \pm 1.12^{\mathrm{a}}$ \\
\hline 40 & Integrin alpha 8 & $1.72 \pm 0.55$ & $3.36 \pm 0.05^{\mathrm{a}}$ \\
\hline 45 & Integrin alpha $\mathrm{x}$ & $1.57 \pm 0.41$ & $3.05 \pm 0.39^{\mathrm{a}}$ \\
\hline 81 & ELAM-1 & $1.31 \pm 0.27$ & $4.26 \pm 0.20^{\mathrm{a}}$ \\
\hline 75 & NCAM & $3.77 \pm 0.71^{\mathrm{a}}$ & $4.40 \pm 1.57^{\mathrm{a}}$ \\
\hline 95 & VCAM-1 & $2.31 \pm 0.30^{\mathrm{a}}$ & $2.95 \pm 0.35^{\mathrm{a}}$ \\
\hline \multicolumn{4}{|c|}{ Extracellular matrix proteins } \\
\hline 20 & Collagen 1a1 & $5.59 \pm 1.45^{\mathrm{a}}$ & $21.73 \pm 2.27^{\mathrm{a}}$ \\
\hline 31 & fibronectin & $2.01 \pm 0.45^{\mathrm{a}}$ & $6.15 \pm 1.04^{\mathrm{a}}$ \\
\hline 54 & Laminin B1 & $1.46 \pm 0.34$ & $2.51 \pm 0.35^{\mathrm{a}}$ \\
\hline 87 & SPARC & $2.65 \pm 0.24^{\mathrm{a}}$ & $3.72 \pm 0.11^{\mathrm{a}}$ \\
\hline 94 & Tenascin $\mathrm{C}$ & $1.20 \pm 0.33$ & $1.87 \pm 0.29^{\mathrm{a}}$ \\
\hline 96 & vitronectin & $1.82 \pm 0.44^{\mathrm{a}}$ & $1,94 \pm 0.68^{\mathrm{a}}$ \\
\hline \multicolumn{4}{|l|}{ Proteases } \\
\hline 1 & Adamts 1 & $1.31 \pm 0.53$ & $4.19 \pm 0.46^{\mathrm{a}}$ \\
\hline 4 & caspase- 8 & $2.35 \pm 0.34^{\mathrm{a}}$ & $1.81 \pm 0.28^{\mathrm{a}}$ \\
\hline 25 & Cathepsin D & $1.80 \pm 0.26^{\mathrm{a}}$ & $1.92 \pm 0.36^{\mathrm{a}}$ \\
\hline 57 & Hyaluronidase & $2.05 \pm 0.45^{\mathrm{a}}$ & $2.31 \pm 0.58^{\mathrm{a}}$ \\
\hline 60 & MMP12 & $1.88 \pm 0.23^{\mathrm{a}}$ & $5.91 \pm 1.20^{\mathrm{a}}$ \\
\hline 67 & MMP2 & $1.10 \pm 0.15$ & $4.15 \pm 1.77^{\mathrm{a}}$ \\
\hline 79 & uPA & $2.88 \pm 0.46^{\mathrm{a}}$ & $3.03 \pm 0.58^{\mathrm{a}}$ \\
\hline \multicolumn{4}{|c|}{ Protease inhibitor } \\
\hline 92 & Timp1 & $9.83 \pm 1.57^{\mathrm{a}}$ & $6.43 \pm 1.78^{\mathrm{a}}$ \\
\hline 93 & Timp2 & $2.25 \pm 0.54^{\mathrm{a}}$ & $4.71 \pm 1.39^{\mathrm{a}}$ \\
\hline
\end{tabular}

Innotech, San Leandro, CA). The relative expression levels were determined after normalization using the signals of GAPDH in the corresponding samples.

In vivo inhibition of integrin $\alpha_{v}$

Mice were treated in vivo with a specific integrin $\alpha_{v}$ inhibitor (EMD 121974, Merck KG, Darmstadt, Germany) 1 day before myocardial infarction. Alzet osmotic mini pumps were implanted subcutaneously. The release rate was $1 \mu \mathrm{l} / \mathrm{h}$ per animal of either a solution of $100 \mathrm{mg} / \mathrm{ml}$ of the integrin $\alpha_{\mathrm{v}}$ inhibitor in 50\% dimethyl sulfoxide (DMSO)/ phosphate-buffered saline (PBS) or DMSO/PBS alone (vehicle control). The inhibitor was characterized previously $[12,13]$ and used by our group to inhibit integrin áv function in smooth muscle cells [14].

Isolation of myocardial cells and cell culture

Cardiac myocytes were isolated and cultured from hearts of 1- to 2-day-old neonatal Wistar rat, as described previously [15]. Isolated cells were pelleted by centrifugation at $1,600 \mathrm{rpm}$ for $5 \mathrm{~min}$ at room temperature, resuspended in complete medium with 5\% fetal bovine serum (FBS), and filtered through a metal sieve. The resulting cells were plated in 6-well dishes or Lab-Tek chamber slides (Nunc Inc., Naperville, IL, USA) at high density $\left(6 \times 10^{5}\right.$ cells/well or $2 \times 10^{5}$ cells/chamber). Mouse embryo fibroblasts were maintained in Dulbecco's Modified Eagle's Medium (DMEM) supplemented with 10\% FBS.

\section{Immunofluorescence staining}

Fresh frozen ventricular tissue sections $(5 \mu \mathrm{m})$ from mouse hearts were mounted on slides, fixed in $4 \%$ paraformaldehyde in Tris-buffered saline (TBS) for $15 \mathrm{~min}$ and treated for 20 min with $1 \%$ Triton X-100 in TBS. After blocking the sections in TBS containing 1\% bovine serum albumin (TBSA), they were incubated overnight with goat antiSPARC antibody (1:100; Santa Cruz Biotechnology, CA sc-13326) in TBSA. The sections were washed and further incubated with Cy2-coupled mouse anti-goat antibodies (Chemicon, Temecula, CA). For actin staining, phalloidinAlexa546 dye (Molecular Probes, Eugene, OR) was used. For nuclear staining, 4', 6-Diamidine-2'-phenylindole dihydrochloride (DAPI) was employed as described earlier [15]. In negative controls, either the first antibody was 
omitted or a SPARC-specific blocking peptide (sc-133269, Santa Cruz Biotechnology) was added at a dilution of 1:10.

\section{ELISA assay}

Secreted transforming growth factor (TGF)- $\beta 1$ and platelet-derived growth factor (PDGF) were quantified using the Quantikine mouse TGF- $\beta 1$ kit and PDGF kit, respectively (R\&D System). SPARC protein was quantified using Osteonectin ELISA kit (US Biological, Swampscott, USA). Cells were processed and assayed according to the manufacturer's protocol. Briefly, serum-starved fibroblasts or cardiac myocytes were cultured on dishes that were plated with VN $(1 \mu \mathrm{g} / \mathrm{ml})$ or poly-L-lysin (ctr, $1 \mu \mathrm{g} / \mathrm{ml})$ as control experiment. As stimulus, TGF- $\beta 1 \quad(5 \mathrm{ng} / \mathrm{ml}$; Calbiochem), or PDGF (5 ng/ml; Calbiochem) was added to the medium for $72 \mathrm{~h}$. After treatment, conditioned medium was collected by centrifugation at $600 \times g$ for $5 \mathrm{~min}$ and stored at $-80^{\circ} \mathrm{C}$ until the assay was performed. Cell numbers were determined, and protein values were normalized to cell numbers.

\section{Cell migration assays}

Millicell modified Boyden chambers $(8-\mu \mathrm{m}$ pore size; Millipore, Bedford, MA) were employed in haptotaxis assays as previously described [16]. Briefly, the lower surface of the Millicell polycarbonate membrane was coated with $100 \mu \mathrm{l}$ DMEM containing the indicated proteins. Specifically, the underside of the membrane was coated with different combinations of FN and/or SPARC (cat. no. 499240; Calbiochem) with or without the specific anti-SPARC antibody (Santa Cruz Biotechnology) at $1 \mu \mathrm{g} / 100 \mathrm{ml}$. Incubation was at room temperature for $30 \mathrm{~min}$. Cells were placed into 24-well plates containing $0.4 \mathrm{ml}$ migration medium or migration medium with $0.5 \%$ FBS. The migratory cells on the lower membrane surface were fixed by methanol/acid treatment and stained with crystal violet $(0.1 \%$ crystal violet, $0.1 \mathrm{M}$ borate $\mathrm{pH} 9.0,2 \%$ ethanol). Haptotaxis was quantified by counting cells in five random fields/chambers using a $20 \times$ objective in three individual chambers per sample. Purity of commercial SPARC preparations was tested with silver staining to rule out contamination with other growth factors (Supplementary Fig. 1).

\section{Statistical analysis}

Analysis of variance (ANOVA) and unpaired $t$ test were used for statistical analysis of the data. A level of $p<0.05$
A

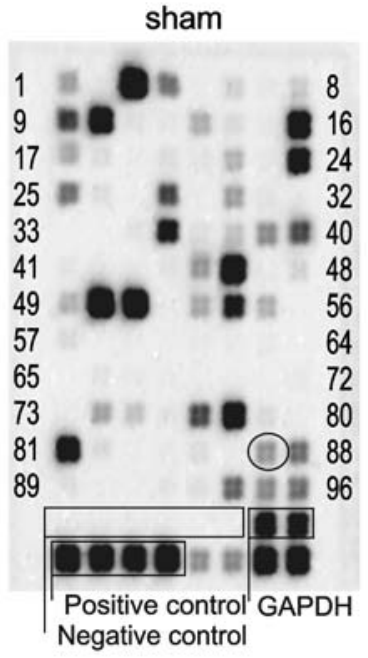

MI-7d

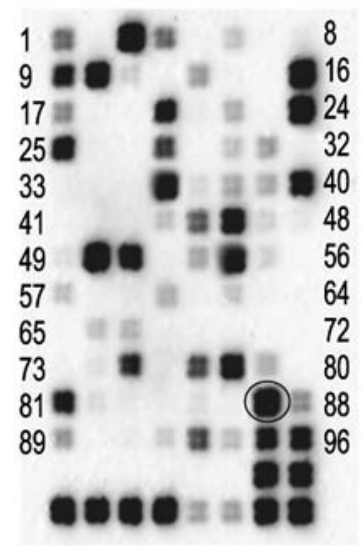

B

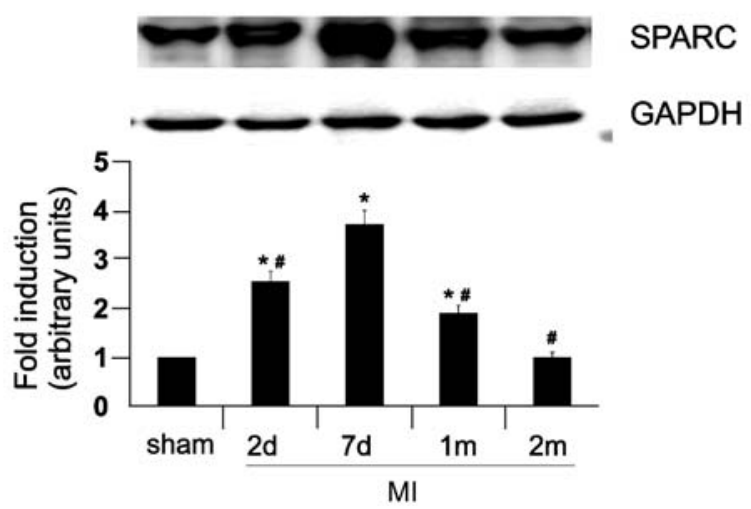

Fig. 1 Gene expression analysis in mouse myocardium 7 days (MI-7d) after experimental myocardial infarction. A total of $112 \mathrm{cDNA}$ fragments (400 bp in length) were examined including 96 spots representing extracellular matrix proteins, integrins, proteases, and related genes. Six spots (Negative control including blanks), four spots (positive control), and the two spots encoding the housekeeping gene glyceraldehyde-3-phosphate dehydrogenase (GAPDH) used for normalization are indicated. All positive controls showed a clear hybridization signal, whereas negative controls consistently showed the absence of any hybridization signal. Membranes were hybridized with RNA isolated from individual animals. For the full layout of the array see supplementary Table 1 . a There are two representative membranes hybridized with RNA isolated from a sham-operated animal and an infarcted animal, respectively. Genes consistently upregulated more than 1.8-fold are shown in Table 1. Circles indicate SPARC. b Changes in SPARC protein levels following myocardial infarction. Representative Western Blot of sham-operated mice (Ctr) or infarcted animals (MI) at the indicated times after the procedure (upper panel). As loading control, samples were probed with antiGAPDH antibodies. The bar diagraph indicates the quantification of SPARC expression. Data are expressed as fold changes from corresponding sham-operated mice and represent means \pm SEM; ${ }^{*} \mathrm{p}<0.01$ vs corresponding sham-operated animals; ${ }^{*} p<0.05$ vs MI-7d 
was accepted as statistically significant. Values are given as mean \pm SEM.

\section{Results}

Gene expression events following experimental myocardial infarction

To analyze gene expression events concerning ECM proteins and the respective cellular receptors and regulators, we compared heart muscle transcript levels of shamoperated animals to the RNA levels observed after experimental myocardial infarction by the help of GEArray cDNA macroarrays. The used arrays encompass 96 cDNAs encoding cell adhesion molecules, ECM proteins, and additional proteins that play central roles in cell-ECM interactions and tissue remodeling (for the layout of the array and the included controls, see Supplementary Table 1). A total of 23 genes were found to be induced more than 1.8-fold 7 days after myocardial infarction (Table 1 and Fig. 1a). As indicators for successful myocardial infarction and induction of cardiac remodeling, collagen and FN were strongly up-regulated [17]. Beside several ECM genes, a prominent induction was observed for the matricellular protein SPARC. Expression of SPARC was significantly increased after 2 days $(2.65 \pm 0.24$-fold, $p<0.01)$ and 7 days $(3.78 \pm 0.11$-fold, $p<0.01)$ following myocardial infarction.

SPARC protein levels are transiently increased during myocardial repair

To verify the results of the cDNA array analysis, we analyzed SPARC protein levels. In line with the observed changes in mRNA levels, SPARC protein levels were strongly increased upon myocardial infarction with a maximal induction at day 7 (Fig. 1b). Within the next 2 months, SPARC protein levels returned to the background levels of sham-operated animals. Probing of the membranes with antibodies against GAPDH demonstrated equal loading of the samples. Quantification of the Western blot signals indicated a significant increase in SPARC protein expression at day 2 (2.58 \pm 0.21 -fold, $p<0.01, n=12)$, day $7(3.72 \pm 0.28$ -

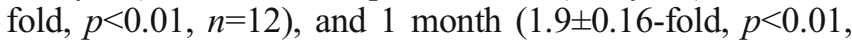
$n=12$ ) following the infarction, respectively (Fig. 1b). Furthermore, the kinetic SPARC expression indicates that this protein presumably plays a transient role during the remodeling of the infarcted heart tissue.
Fig. 2 SPARC deposition in the infarcted heart. Tissue sections were stained for SPARC, actin, and DAPI (nuclei) in normal heart and after myocardial infarction (MI). SPARC is barely detectable in normal myocardium (ctr). To prove for specificity of SPARC staining, 7 days after myocardial infarction there was no SPARC immunoreactivity in presence of a SPARC blocking peptide. However, 7 days after MI (MI-7d), immunoreactivity for SPARC is enhanced (without a blocking peptide) in the infarcted area, while the actin signal is reduced, indicating the necrotic infarcted area. DAPI staining noted a large number of nuclei in the infarcted area (MI-7d). Two months after MI, there is no significant SPARC staining in the scar area (MI-2 m)
SPARC
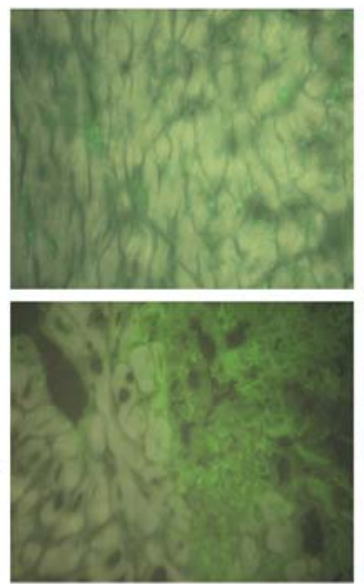

MI-7d

blocking peptide

MI-7d
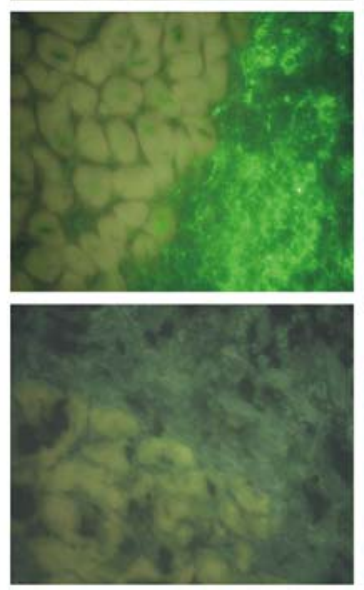

nuclei
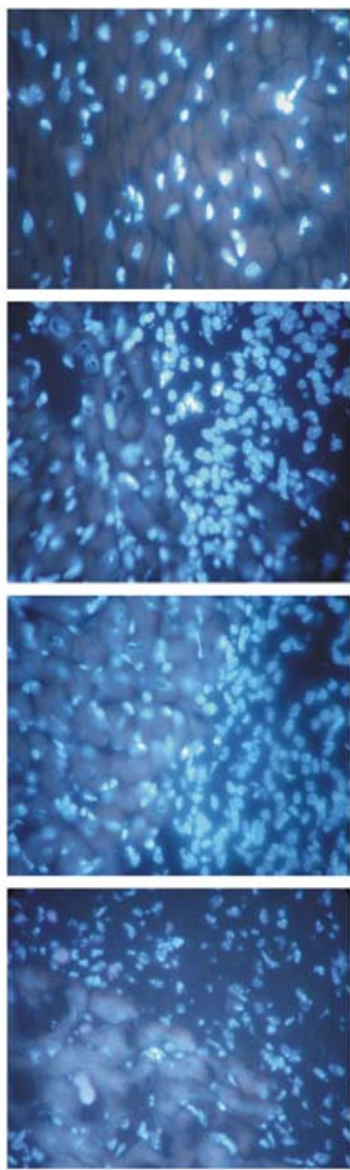

actin
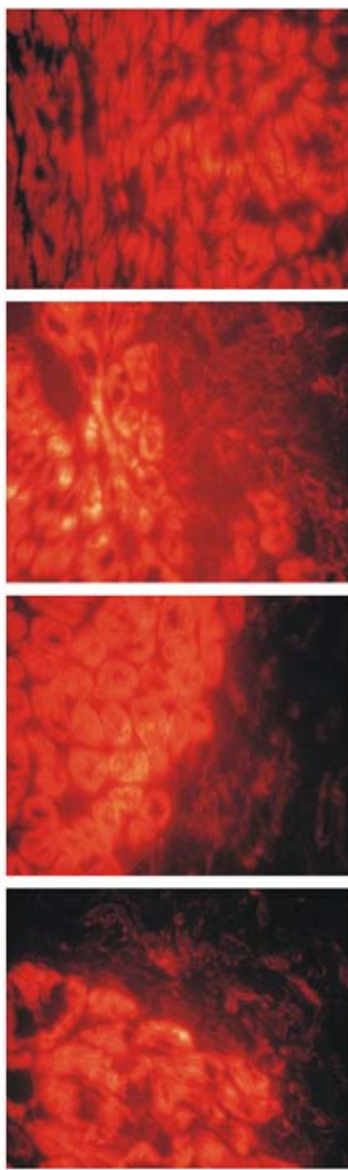
Enhanced SPARC expression dominates in the infarcted area

To analyze the tissue distribution of SPARC after myocardial infarction, frozen sections of infarcted tissue or control mouse hearts were stained with anti-SPARC antibodies and detected with fluorescence-labeled secondary antibodies. To identify both cardiac myocytes and nonmyocytes, cellular actin and nuclei were also labeled with phalloidin-rhodamine and DAPI, respectively (Fig. 2). To test for specificity of the SPARC antibody used, either the SPARC antibody was omitted (data not shown) or a blocking peptide (encompassing the region used to generate the SPARC antibody) was included. No significant SPARC staining could be observed in the negative controls ("blocking peptide" in Fig. 2). Importantly, the expression of SPARC was low in normal heart tissue (Fig. 2). However, 2 days after infarction, significant increases in SPARC expression could be observed in the infarcted area. Similarly, a decrease in the phalloidin staining, suggestive of actin degradation in the infarcted zone, together with an increased number of nuclei, suggestive of invasion of inflammatory cells and fibroblasts, could be observed. Moreover, 7 days after the infarction, strong SPARC staining was evident with further reduction in the actin signal and a strongly increased number of nuclei (presumably nonmyocytes). SPARC deposition was localized dominantly to the infarcted area surrounding the invaded fibroblasts and inflammatory cells. Strikingly, 2 months after the experimental infarction, SPARC immunoreactivity in the infarcted area was decreased again to the background levels seen in normal heart tissue, while an organized scar tissue without actin staining and elevated nuclear signal from fibroblasts and other nonmyocytes could be seen. These data demonstrated that enhanced expression of SPARC is transient in the remodeling tissue. Furthermore, up-regulation of SPARC coincides with the invasion of fibroblasts and the induction of scar formation in the injured heart tissue.

Induction of SPARC depends on integrin $\alpha_{\mathrm{v}}$ function

Previously, we have observed a prominent role of integrins in modulating tissue remodeling events upon myocardial infarction $[18,19]$. Therefore, mice that underwent myocardial infarction were simultaneously treated with a specific integrin $\alpha_{\mathrm{v}}$ inhibitor or the vehicle control. Gene expression events observed in infarcted animals treated
Fig. 3 Inhibition of integrin $\alpha_{v}$ blocks SPARC secretion after myocardial infarction (MI). a SPARC mRNA expression in mouse hearts 7 days after MI without (MI-7d, left) and with (MI-Inh-7d, right) $\alpha_{\mathrm{v}}$ integrin inhibitor. Circles indicate SPARC. b SPARC protein expression in mouse hearts 2 and 7 days after MI. Application of the $\alpha_{v}$ integrin inhibitor via mini pumps blocked the increase in SPARC protein expression. Data are expressed as fold changes from corresponding sham-operated mice, normalized to GAPDH. Values are mean \pm SEM; $n=12 ; * p<0.01 \mathrm{ctr}$ vs sham-operated animals; ${ }^{*} p<0.05$ MI vs MI-Inh
A
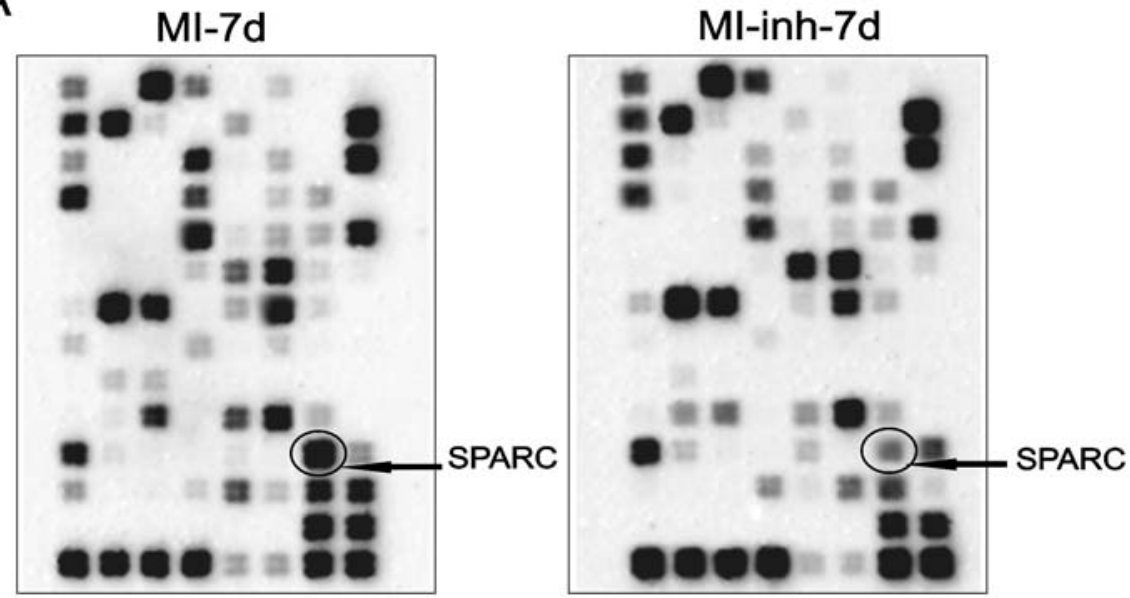

B
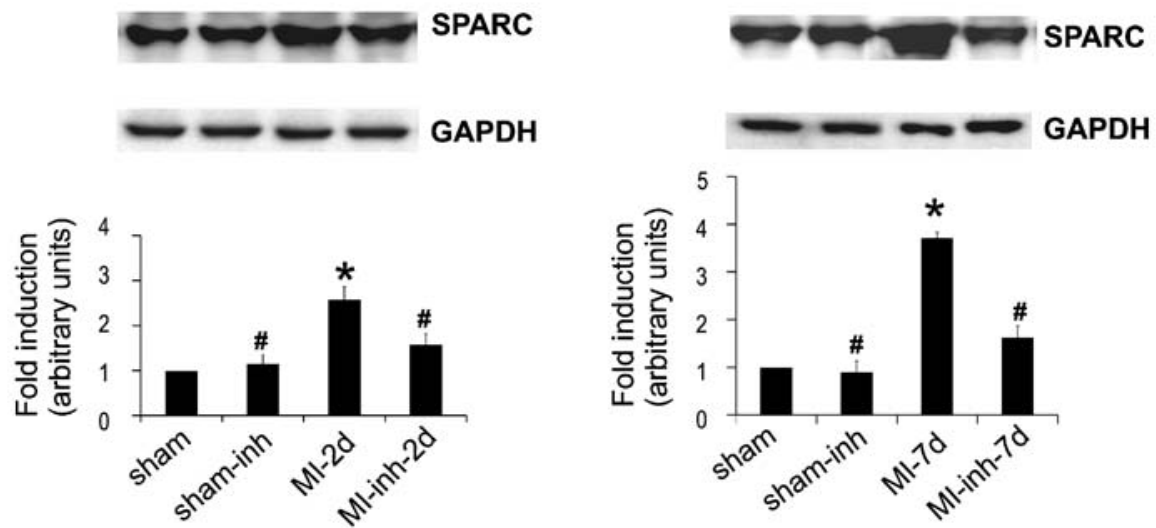
with the vehicle control were identical to those observed previously upon myocardial infarction, and the same set of genes was found to be up-regulated after 7 days (Fig. 3a). Importantly, up-regulation of SPARC expression following myocardial infarction was dependent on integrin $\alpha_{\mathrm{v}}$ function as the inhibitor blocked the increase in SPARC mRNA at day 2 (1.42 \pm 0.37 -fold) and day 7 (1.64 \pm 0.29 fold) (Fig. 3a) after myocardial infarction. This was in contrast to the $2.58 \pm 0.24$-fold and $3.78 \pm 0.11$-fold induction of SPARC mRNA 2 and 7 days after infarction in the vehicle control group, respectively. Again, the up-regulation of SPARC mRNA levels was closely followed by similar increases in SPARC protein expression (Fig. 3b). Inhibition of integrin $\mathrm{a}_{\mathrm{v}}$ severely blocked the increase in SPARC protein 2 days $(1.58 \pm 0.24$-fold vs $2.58 \pm 0.21$-fold, $p>0.01, n=12)$ and 7 days $(1.63 \pm 0.22$-fold vs $3.72 \pm 0.28$ fold, $p>0.01, n=12$ ) after infarction (Fig. 3b).

Induction of SPARC by TGF- $\beta 1$ and PDGF in fibroblasts and cardiac myocytes

To investigate the cellular origin of SPARC and the role of integrin $\alpha_{\mathrm{v}}$ in this process, we stimulated isolated cardiac myocytes and fibroblast with VN. To our surprise, VN, a well-characterized ligand for integrin $\alpha_{v}$, had no effect on SPARC expression in cardiac myocytes or fibroblasts (Fig. 4b). However, SPARC expression in isolated cardiac myocytes could be strongly stimulated by TGF- $\beta 1(2.8 \pm$ 0.1 -fold, $p<0.01)$ and PDGF $(2.3 \pm 0.3$-fold, $\quad p<0.01$; Fig. 4a). Similarly, treatment of fibroblasts with either TGF- $\beta 1$ (3.9 \pm 0.26 -fold, $p<0.01)$ or PDGF $(3.2 \pm 0.30$-fold, $p<0.01)$ significantly increased SPARC expression as compared to untreated cells (ctr, Fig. 4b). Consistent with the failure of VN to stimulate SPARC expression, inhibition of integrin $\alpha_{\mathrm{v}}$ did not interfere with TGF- $\beta 1$ or PDGFstimulated SPARC secretion as determined by ELISA assays (Fig. 4c,d). SPARC secretion by fibroblasts in response to TGF- $\beta 1$ or PDGF was considerably higher (TGF- $\beta 1: 855 \mathrm{ng} / \mathrm{ml} \pm 46$, PDGF: $721 \mathrm{ng} / \mathrm{ml} \pm 44$ ) than the levels of SPARC secreted by cardiac myocytes (TGF- $\beta 1$ : $220 \mathrm{ng} / \mathrm{ml} \pm 33$, PDGF: $189 \mathrm{ng} / \mathrm{ml} \pm 25$; Fig. 4c,d). These results suggested that the effect of integrin $\alpha_{v}$ on SPARC expression in vivo might be indirect, and integrin $\alpha_{v}$ might function upstream from growth-factor-mediated stimulation of SPARC expression.

VN stimulates TGF- $\beta 1$ and PDGF expression in cardiac myocytes

To investigate if integrin $\alpha_{v}$ engagement might indirectly make an impact on SPARC gene expression by modulating the secretion of growth factors, we studied TGF- $\beta 1$ and PDGF expression in cardiac myocytes after stimulation with VN. We observed that activation of isolated cardiac myocytes with VN $(1 \mu \mathrm{g} / \mathrm{ml})$ significantly increased TGF- $\beta 1(423 \pm 115 \mathrm{pg} / \mathrm{ml} ; n=12, p<0.05)$ and PDGF $(84 \pm$ $17 \mathrm{pg} / \mathrm{ml} ; n=12, p<0.05$ ) expression (Fig. 5a,b). This increase was completely abrogated by the addition of an integrin $\alpha_{\mathrm{v}}$ inhibitor (Fig. 5a,b). Interestingly, no significant stimulation of TGF- $\beta 1$ and PDGF expression by VN was observed in fibroblasts (data not shown). Together, these data with isolated cells suggested that in vivo, a cross
Fig. 4 SPARC expression in cardiac myocytes and fibroblasts. a Cultured cardiac myocytes were stimulated with TGF- $\beta 1$ ( $5 \mathrm{ng} / \mathrm{ml})$ or PDGF-BB $(5 \mathrm{ng} / \mathrm{ml})$. Growth factors induced a significant increase in SPARC protein expression. SPARC concentration was assessed in the culture medium $72 \mathrm{~h}$ after stimulation. b Same as panel $\mathbf{a}$ in fibroblasts. Vitronectin (VN) stimulation did not cause SPARC secretion. c ELISA for SPARC in neonatal rat cardiac myocytes. SPARC expression in cardiac myocytes increased after TGF or PDGF treatment. Inhibition of integrin $\alpha_{v}$ did not decrease SPARC expression significantly. d Same as panel $\mathbf{c}$ in fibroblast cell cultures. Please note that there is a significantly higher level of absolute values of SPARC secreted from fibroblasts, compared to cardiac myocytes. Values are the mean \pm SEM, $* P<0.05$ vs untreated cardiac myocytes $(n=12)$
A
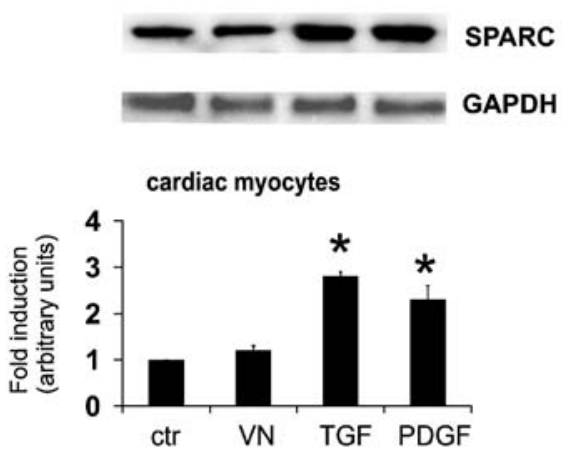

\section{C}

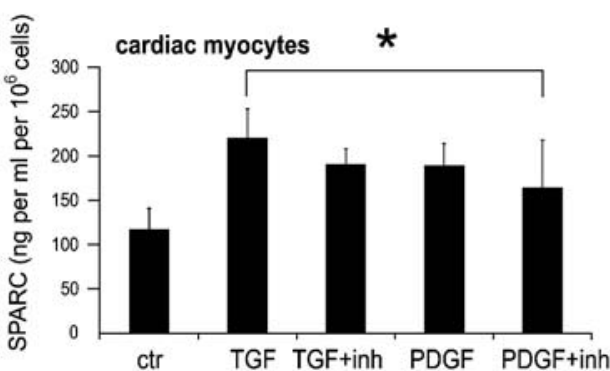

B
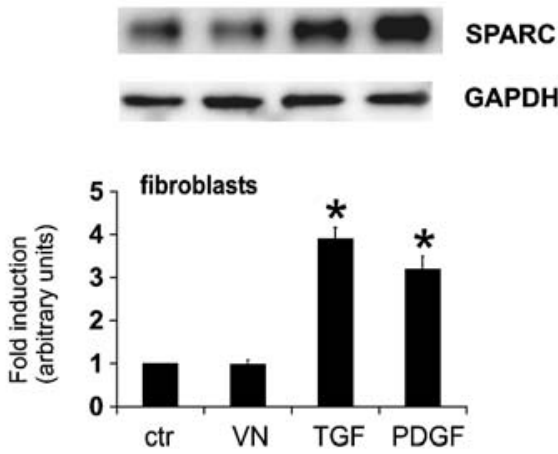

D

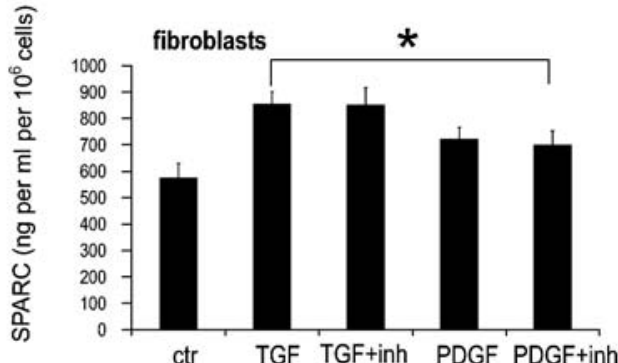


A

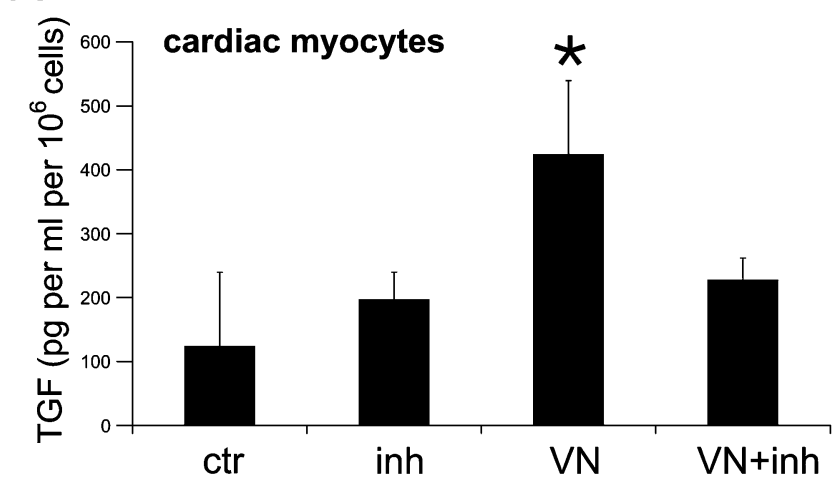

B

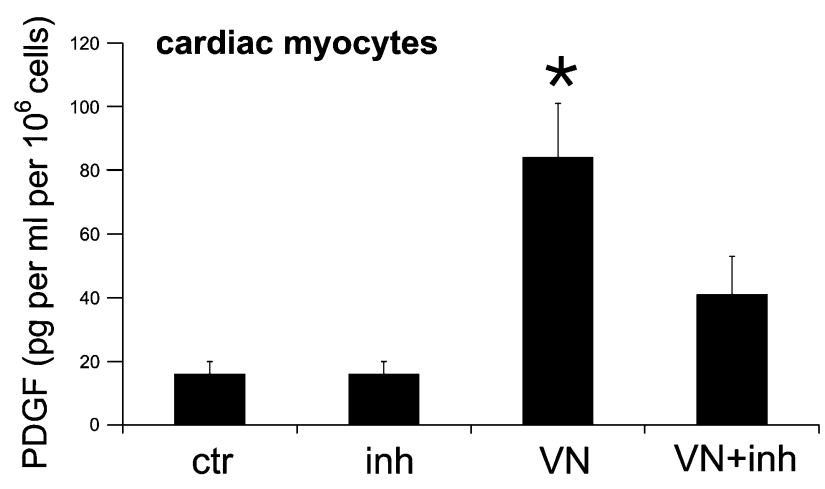

Fig. 5 Growth factor expression from cardiac myocytes. Isolated cardiac myocytes were cultured on dishes, coated with poly-Llysin (ctr) of vitronectin ( $\mathrm{VN}$, an integrin $\alpha_{\mathrm{v}}$ ligand). TGF- $\beta 1$ and PDGF$\mathrm{BB}$ concentrations were determined in the supernatant of the cells. Concentration was determined using ELISA assays. There is a significant up-regulation of $\mathbf{a}$ TGF and $\mathbf{b}$ PDGF in cardiac myocytes after stimulation with vitronectin. Simultaneous addition of an integrin $\alpha_{\mathrm{v}}$ inhibitor (Inh) suppressed the increase in growth factor expression. Therefore, secretion of TGF and PDGF from cardiac myocytes is highly dependent on integrin $\alpha_{\mathrm{v}}$ function. ${ }^{*} P<0.05$ vs $\operatorname{ctr}(n=12)$

talk between different cell types might finally lead to SPARC secretion and that the initial VN-induced growth factor release from cardiac myocytes might promote strong SPARC expression predominantly by fibroblasts.

SPARC modulates the haptotaxis response of fibroblasts toward FN

To get an idea about the functional significance of SPARC during wound healing, we analyzed the haptotaxis response of fibroblasts on a FN matrix in the presence or absence of SPARC. Haptotaxis is the directed movement of cells induced by a gradient of an immobilized stimulus such as an ECM protein. It is used as a term to distinguish this migration from chemotaxis that is induced by soluble stimuli.

To this end, modified Boyden migration chambers were coated on the underside of the membrane with different combinations of FN and SPARC. Fibroblasts seeded into the top compartment were allowed to migrate for $7 \mathrm{~h}$. Clearly, FN-coated chambers stimulated a dose-dependent migratory response, whereas in control chambers, where the membrane had been coated with BSA, fibroblasts did not migrate from the top to the bottom compartment (Fig. 6a). Interestingly, coating with a combination of FN and a constant amount of SPARC $(1 \mu \mathrm{g} / \mathrm{ml})$ led to an increased haptotaxis response for each $\mathrm{FN}$ concentration tested (Fig. 6a,c). The effect of SPARC could be blocked by adding a polyclonal anti-SPARC antibody, demonstrating the specificity of SPARC-mediated enhancement of FN-stimulated migration.

It is important to note that SPARC, on its own at concentrations ranging from 1 to $10 \mu \mathrm{g} / \mathrm{ml}$, did not stimulate cell motility (Fig. 6b). Again, a combination of FN $(1 \mu \mathrm{g} /$ $\mathrm{ml})$ and low concentrations of SPARC $(1 \mu \mathrm{g} / \mathrm{ml})$ enhanced FN-stimulated fibroblast migration (Fig. 6b). In contrast, when higher SPARC concentrations $(5-10 \mu \mathrm{g} / \mathrm{ml})$ were combined with a given concentration of FN $(1 \mu \mathrm{g} / \mathrm{ml})$, the promigratory activity of SPARC was reversed and an inhibitory effect of SPARC was observed (Fig. 6b,c). This inhibitory effect of SPARC at higher concentrations could be overcome by addition of $0.5 \% \mathrm{FBS}$, demonstrating that the used commercial preparation of SPARC was not contaminated by general inhibitors of cell migration (Fig. 6b). These results provide evidence that SPARC can modulate ECM-triggered haptotaxis of fibroblasts. Furthermore, these data are in line with the idea that SPARC acts as a switch to first promote, and then at higher concentrations, to shut off fibroblast migration during tissue remodeling events.

\section{Discussion}

Expression and abundance of ECM proteins and their cellular receptors undergo dramatic changes following myocardial infarction to preserve organ function. In the present study, we analyzed gene expression changes upon experimental myocardial infarction in the mouse and followed these changes in the course of the healing response up to 2 months after infarction. Most strikingly, we observed a transient increase in SPARC expression in response to myocardial infarction. Induction of SPARC was dependent in part on integrin $\alpha_{\mathrm{v}}$ function. In vitro, enhanced SPARC expression could be demonstrated mainly for fibroblasts but also for cardiac myocytes, e.g., if these cells were stimulated with TGF- $\beta 1$ and PDGF. As SPARC modulated the FNstimulated migration of fibroblasts, our results pointed to an important role of SPARC in the transient healing response after myocardial infarction.

Several studies have indicated that SPARC expression is high during embryonic development, but usually low during normal postnatal life. However, SPARC can be induced in various tissues such as bone, gut mucosa, and skin during healing responses $[20,21]$. In this context, SPARC may contribute to the reorganization of connective tissue and stimulate angiogenesis [22, 23]. It was already demonstrated, that the integrin inhibitor (EMD 121974) used in this study was able to inhibit angiogenesis [24]. With respect to heart tissue, SPARC is abundantly ex- 
A

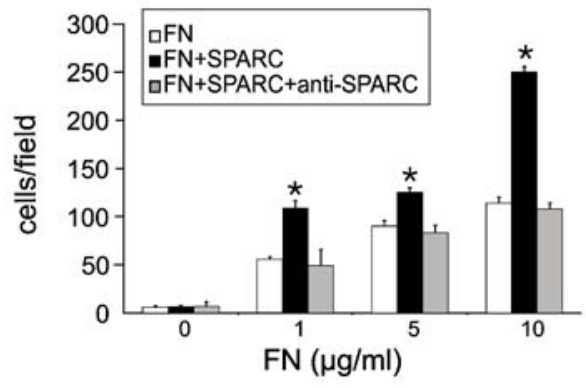

C

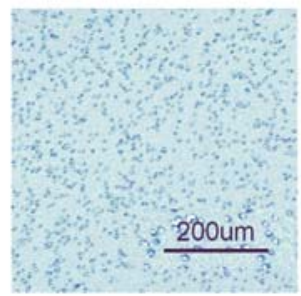

BSA
B

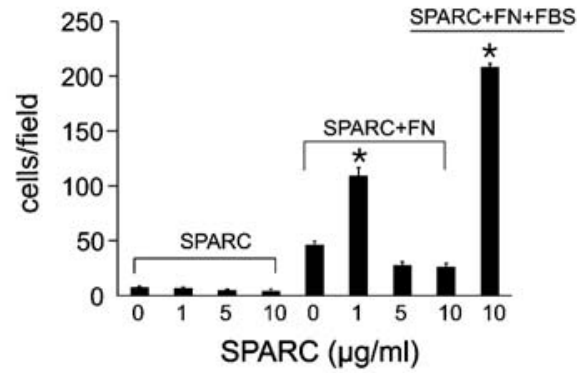

Fig. 6 SPARC modulates fibronectin-stimulated haptotaxis. a Serumstarved fibroblasts were employed in haptotaxis assays using modified Boyden migration chambers coated with fibronectin (FN) at the indicated concentrations in the absence (open bar) or the presence of SPARC $(1 \mu \mathrm{g} / \mathrm{ml}$, closed bar $)$. There is a significant increase of fibroblast migration with rising fibronectin concentrations. Addition of an anti-SPARC antibody (gray bars) abolishes effects of SPARC on FN-induced cell migration. b Haptotaxis of fibroblasts was analyzed in
Boyden chambers coated with SPARC at the indicated concentrations in the absence (open bar) or the presence of FN $(1 \mu \mathrm{g} / \mathrm{ml}$; closed bar). Whereas low concentrations of SPARC enhance FN-initiated haptotaxis, higher SPARC concentrations have an inhibitory effect. Addition of FBS $(0.5 \%)$ overcomes the inhibitory effect of high amounts of SPARC, demonstrating that purified SPARC is not contaminated with general inhibitors of migration. $\mathbf{c}$ Images show representative fields of view from the indicated samples pressed in this organ during early development. In contrast, its expression in the heart is very low during adulthood [20, 22]. Importantly, increased mRNA levels of SPARC have been detected in the myocardium following myocardial infarction [5, 17], indicating a role for SPARC in matrix remodeling during wound healing in the heart.

One important property of SPARC is its anti-adhesive effect. Accordingly, SPARC can modulate the interactions between cells and their surrounding matrix by inhibiting adhesion and spreading on a collagen matrix [25]. In the process of repair after myocardial infarction, necrotic cells in the infarcted area are gradually absorbed and replaced by scar tissue. Scar formation is already evident within a few days after infarction, and the scar tissue is characterized mainly by a collagen- and FN-rich ECM and the presence of fibroblasts (Fig. 7). Our gene expression analysis demonstrates that SPARC is co-regulated together with several ECM proteins including collagen, FN, and laminin, and their enhanced transcription is detectable within 2 days after infarction. By immunostaining, the increased SPARC protein levels were mainly localized to the infarcted, necrotic tissue during the initial healing response. This early increase in SPARC might help to loosen the strong adhesive connections between surviving cardiac myocytes and connective tissue, thereby allowing the invasion of fibroblasts, facilitating tissue reorganization and scar formation.
The molecular mechanism that connects myocardial infarction with increased SPARC expression in vivo is currently unknown. To this point, our results clearly establish an important role for integrin $\alpha_{\mathrm{v}}$ in the regulation of SPARC in vivo, as SPARC mRNA levels were severely compromised in infarcted animals receiving a specific integrin $\alpha_{\mathrm{v}}$ inhibitor.

However, stimulation of isolated cardiac myocytes or fibroblasts with $\mathrm{VN}$, a well-characterized ligand for integrin $\alpha_{v} \beta_{3}$ and $\alpha_{v} \beta_{5}$, failed to induce SPARC secretion in vitro, suggesting that integrin $\alpha_{\mathrm{v}}$ has an indirect role in the upregulation of this matricellular protein. Our further experiments point to growth factors such as TGF- $\beta 1$ and PDGF that moderately induce SPARC secretion in isolated rat neonatal cardiac myocytes, but very strongly in fibroblasts. In addition, these growth factors have been shown to increase SPARC expression in human gingival fibroblasts, rabbit articular chondrocytes, and human pulp cells [26-29].

Growth factor cDNAs were not included on the GEArray, and therefore, their regulation was not examined after myocardial infarction in the present study. Because of this, the level of TGF- $\beta 1$ is strongly enhanced in infarcted compared to noninfarcted myocardium within 2 days after coronary artery ligation in the rat $[30,31]$. The measurement of TGF, PDGF, and SPARC secreted from isolated cells in vitro suggests a coordinated sequence of events 


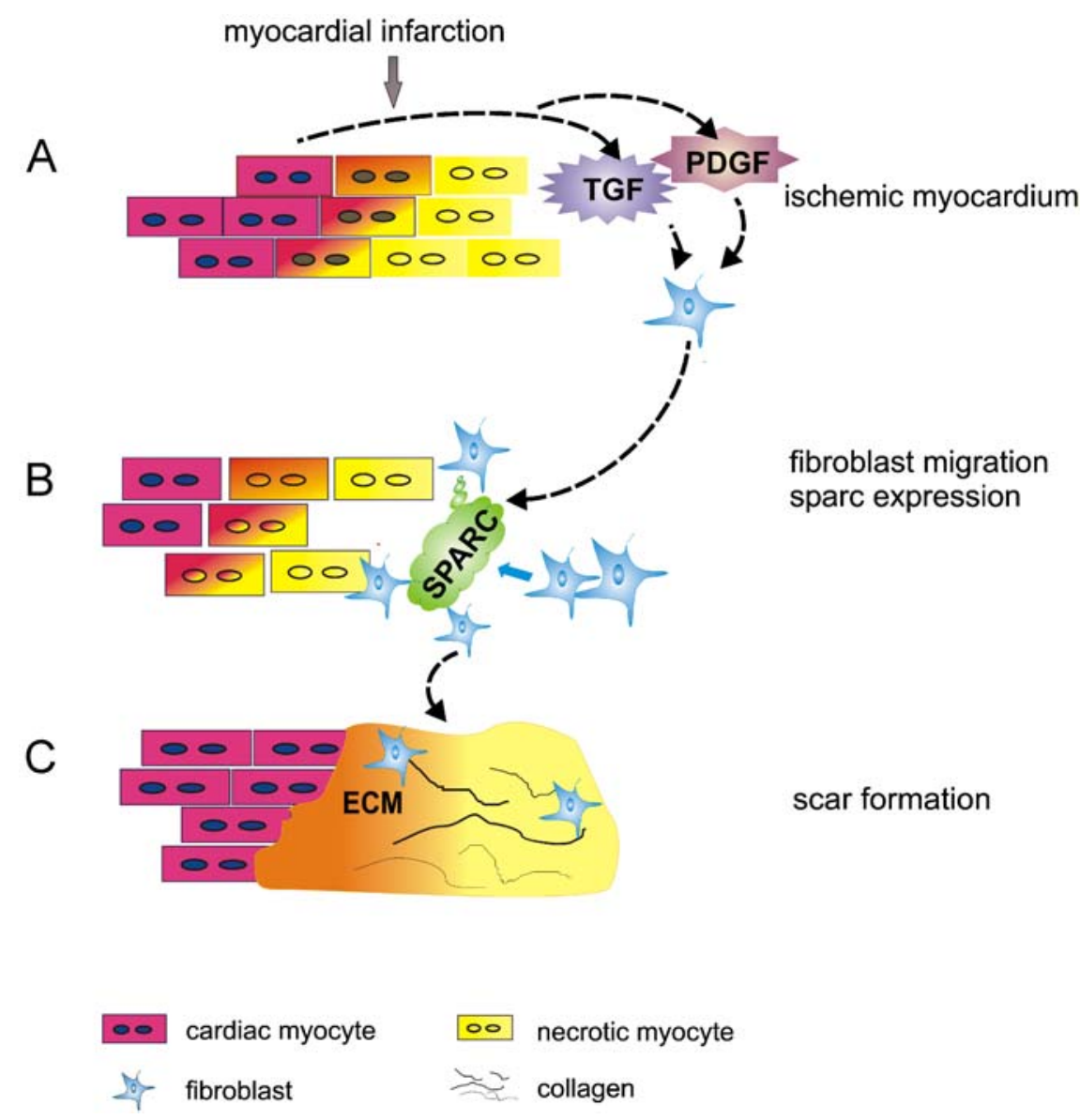

Fig. 7 Schematic drawing summarizing wound healing processes after myocardial infarction (MI) with special regard to SPARC expression and fibroblast migration. a As a result of myocardial ischemia there is an increased expression of growth factors (e.g., TGF and PDGF) secreted from cardiac myocytes. These growth factors mainly act on fibroblasts. b Activated by TGF/PDGF fibroblasts increase SPARC expression. SPARC may open up spaces for the invading fibroblasts (possibly by activating MMP's) and simultaneously act as haptotactic stimulus to promote further fibroblast migration. c Fibroblasts at high density in the scar

eventually cause wound healing and scar formation after myocardial infarction with deposition of collagen and reorganization of the extracellular matrix. In our model, SPARC not only contributes to the initial recruitment of fibroblasts to the infarcted area, but at higher concentrations also helps to limit fibroblast influx. SPARC concentration decreases gradually over time in the scar area, accompanied by a decrease in the number of fibroblasts in the scar. $T G F$ transforming growth factor, $P D G F$ platelet derived growth factor, $E C M$ extracellular matrix

where, in a first step, integrin $\alpha_{\mathrm{v}}$ engagement on cardiac myocytes causes an up-regulation of TGF and PDGF. Inhibition of integrin $\alpha_{v}$ can abrogate this VN-mediated increase in growth factor secretion. In a second step, we envision that SPARC expression is increased moderately after TGF and PDGF stimulation of cardiac myocytes in an autocrine manner. At the same time, the growth factors secreted by cardiac myocytes promote a strong increase in SPARC expression by fibroblasts. Such a two-step process that requires communication between different cell types would also help to explain why specific integrin $\alpha_{\mathrm{v}}$ inhibition that severely interfered with SPARC expression in vivo did not block growth factor-induced SPARC expression in vitro. Furthermore, it is also a key to understand the failure of $\mathrm{VN}$ to directly stimulate a significant level of SPARC expression by isolated cardiac myocytes or fibroblasts.

Together, these observations indicate a complex interplay between growth factors, integrins, and the induction of

ECM and matricellular proteins that mediate the healing response in the infarcted heart. Recent work has also demonstrated that SPARC itself can up-regulate TGF- $\beta 1$, and this up-regulation accounts for the secretion of collagen I in different cell types [32, 33]. Most importantly, our results provide novel insight into the functional role of SPARC in cell migration induced by immobilized ECM proteins. Clearly, SPARC alone did not serve as a haptotactic stimulus for fibroblasts. (Haptotaxis is the directed movement of cells induced by a gradient of an immobilized stimulus such as an ECM protein.) However, in combination with $\mathrm{FN}$, low concentrations of SPARC $(1 \mu \mathrm{g} / \mathrm{ml})$ consistently elevated the promigratory effect of FN. These results are in line with previous reports that have indicated a positive effect of SPARC on FN-induced cell migration. Surprisingly, increases in SPARC concentration in the context of constant amounts of FN resulted in an impairment of FN-stimulated haptotaxis. These data suggest that the balance between SPARC and FN deter- 
mines the effect of these proteins on cell migration. Indeed, cell migration on ECM substrates requires an optimum balance between adhesion and detachment to allow efficient movement of adherent cells. The coexistent effects of SPARC on promotion and inhibition of migration have been also observed in a glioma cell line [34]. As SPARC modulates cellular adhesion to the ECM by direct interaction with several ECM proteins [35], the concentration of SPARC and, more importantly, the ratio between SPARC and its binding partners in the ECM might influence fibroblast migration in a positive or negative way. It is interesting to speculate in this context that the transient accumulation of SPARC in the infarcted zone might initially contribute to enhanced fibroblast migration into this area, whereas later, with higher SPARC concentrations, further fibroblast migration will be limited. Clearly, studies with SPARC-deficient mice have demonstrated that this protein is required for a proper wound healing response and efficient cell migration in skin [36], and this is consistent with our findings in the mouse heart after experimental myocardial infarction.

Together, our combined in vivo and in vitro data suggest an important modulatory role for the matricellular protein SPARC in the healing response following myocardial infarction. Deposition of SPARC in the infarcted zone seems to be intimately involved in scar formation, presumably by promoting the migration of fibroblasts. Whether the transient up-regulation of SPARC after myocardial infarction is essential for the maintenance of cardiac structure and function requires further investigation.

Acknowledgements This work was supported by grants to OR by the German Research Foundation (DFG Ri 1085/3-1) and to OR and ML and CRH by the Interdisciplinary Centre for Clinical Research Würzburg [IZKF E-25(1) and Z-4/47], respectively.

Rong Xue $\mathrm{Wu}$ and Martin Laser contributed equally to this work.

\section{References}

1. Shai SY et al (2002) Cardiac myocyte-specific excision of the beta 1 integrin gene results in myocardial fibrosis and cardiac failure. Circ Res 90(4):458-464

2. Schellings MW, Pinto YM, Heymans S (2004) Matricellular proteins in the heart: possible role during stress and remodeling. Cardiovasc Res 64(1):24-31

3. Bradshaw AD, Reed MJ, Sage EH (2002) SPARC-null mice exhibit accelerated cutaneous wound closure. J Histochem Cytochem 50(1):1-10

4. Funk SE, Sage EH (1993) Differential effects of SPARC and cationic SPARC peptides on DNA synthesis by endothelial cells and fibroblasts. J Cell Physiol 154(1):53-63

5. Komatsubara I et al (2003) Spatially and temporally different expression of osteonectin and osteopontin in the infarct zone of experimentally induced myocardial infarction in rats. Cardiovasc Pathol 12(4):186-194

6. Masson S et al (1998) Remodelling of cardiac extracellular matrix during beta-adrenergic stimulation: upregulation of SPARC in the myocardium of adult rats. J Mol Cell Cardiol 30(8):1505-1514

7. Simkhovich BZ et al (2003) Gene expression profiling - a new approach in the study of myocardial ischemia. Cardiovasc Pathol 12(4):180-185
8. Bornstein P, Sage EH (2002) Matricellular proteins: extracellular modulators of cell function. Curr Opin Cell Biol 14 (5):608-616

9. Lane TF, Sage EH (1994) The biology of SPARC, a protein that modulates cell-matrix interactions. FASEB J 8(2):163-173

10. De S et al (2003) Molecular pathway for cancer metastasis to bone. J Biol Chem 278(40):39044-39050

11. Sturm RA et al (2002) Osteonectin/SPARC induction by ectopic beta(3) integrin in human radial growth phase primary melanoma cells. Cancer Res 62(1):226-232

12. Lode HN et al (1999) Synergy between an antiangiogenic integrin alphav antagonist and an antibody-cytokine fusion protein eradicates spontaneous tumor metastases. Proc Natl Acad Sci USA 96(4):1591-1596

13. DeNardo SJ et al (2000) Neovascular targeting with cyclic RGD peptide (cRGDf-ACHA) to enhance delivery of radioimmunotherapy. Cancer Biother Radiopharm 15(1):71-79

14. Varadarajulu $\mathrm{J}$ et al (2005) Targeting of alpha(v) integrins interferes with FAK activation and smooth muscle cell migration and invasion. Biochem Biophys Res Commun 331 (2):404-412

15. Burkard N et al (2005) Targeted proteolysis sustains calcineurin activation. Circulation 111(8):1045-1053

16. Hauck CR et al (2001) Inhibition of focal adhesion kinase expression or activity disrupts epidermal growth factorstimulated signaling promoting the migration of invasive human carcinoma cells. Cancer Res 61(19):7079-7090

17. Stanton LW et al (2000) Altered patterns of gene expression in response to myocardial infarction. Circ Res 86(9):939-945

18. Laser $M$ et al (2000) Integrin activation and focal complex formation in cardiac hypertrophy. J Biol Chem 275(45): 35624-35630

19. Ross RS (2002) The extracellular connections: the role of integrins in myocardial remodeling. J Card Fail 8[6 Suppl]: S326-S331

20. Holland PW et al (1987) In vivo expression of mRNA for the Ca++-binding protein SPARC (osteonectin) revealed by in situ hybridization. J Cell Biol 105(1):473-482

21. Sage H et al (1989) SPARC, a secreted protein associated with cellular proliferation, inhibits cell spreading in vitro and exhibits $\mathrm{Ca}+2$-dependent binding to the extracellular matrix. J Cell Biol 109(1):341-356

22. Sage $\mathrm{H}$ et al (1989) Distribution of the calcium-binding protein SPARC in tissues of embryonic and adult mice. J Histochem Cytochem 37(6):819-829

23. Reed MJ et al (1993) Differential expression of SPARC and thrombospondin 1 in wound repair: immunolocalization and in situ hybridization. J Histochem Cytochem 41(10):1467-1477

24. Nisato RE et al (2003) Alphav beta 3 and alphav beta 5 integrin antagonists inhibit angiogenesis in vitro. Angiogenesis 6 (2): 105-119

25. Sage EH, Bornstein P (1991) Extracellular proteins that modulate cell-matrix interactions. SPARC, tenascin, and thrombospondin. J Biol Chem 266(23):14831-14834

26. Wrana JL, Overall CM, Sodek J (1991) Regulation of the expression of a secreted acidic protein rich in cysteine (SPARC) in human fibroblasts by transforming growth factor beta. Comparison of transcriptional and post-transcriptional control with fibronectin and type I collagen. Eur J Biochem 197 (2):519-528

27. Zhou H et al (1993) Differential effects of transforming growth factor-beta 1 and bone morphogenetic protein 4 on gene expression and differentiated function of preosteoblasts. J Cell Physiol 155(1):112-119

28. Reed MJ et al (1994) TGF-beta 1 induces the expression of type I collagen and SPARC, and enhances contraction of collagen gels, by fibroblasts from young and aged donors. J Cell Physiol 158(1):169-179 
29. Shiba H et al (1998) Differential effects of various growth factors and cytokines on the syntheses of DNA, type I collagen, laminin, fibronectin, osteonectin/secreted protein, acidic and rich in cysteine (SPARC), and alkaline phosphatase by human pulp cells in culture. J Cell Physiol 174(2):194-205

30. Thompson NL et al (1988) Transforming growth factor beta-1 in acute myocardial infarction in rats. Growth Factors 1(1):91-99

31. Deten A et al (2001) Changes in extracellular matrix and in transforming growth factor beta isoforms after coronary artery ligation in rats. J Mol Cell Cardiol 33(6):1191-1207

32. Francki A et al (2004) SPARC regulates TGF-beta1-dependent signaling in primary glomerular mesangial cells. J Cell Biochem 91(5):915-925
33. Schiemann BJ, Neil JR, Schiemann WP (2003) SPARC inhibits epithelial cell proliferation in part through stimulation of the transforming growth factor-beta-signaling system. Mol Biol Cell 14(10):3977-3988

34. Rempel SA et al (2001) SPARC modulates cell growth, attachment and migration of U87 glioma cells on brain extracellular matrix proteins. J Neurooncol 53(2):149-160

35. Brekken RA, Sage EH (2000) SPARC, a matricellular protein: at the crossroads of cell-matrix. Matrix Biol 19(7):569-580

36. Basu A et al (2001) Impaired wound healing in mice deficient in a matricellular protein SPARC (osteonectin, BM-40). BMC Cell Biol 2(1):15 